\title{
A New CVSS-Based Tool to Mitigate the Effects of Software Vulnerabilities
}

\author{
Assad Ali, Pavol Zavarsky, Dale Lindskog, Ron Ruhl \\ Information Systems Security Management \\ Concordia University College of Alberta, Edmonton,T5B 4E4 Canada
}

\begin{abstract}
The organizations are challenged by the number of vulnerabilities in the software and hardware platforms. Successful execution of the operations need to have vulnerabilities clean environment. The U.S. National Vulnerability Database (NVD) uses Common Vulnerability Scoring System (CVSS) to score each vulnerability found and provides the detailed description of those security vulnerabilities. The score provided by the NVD is based on the intrinsic and the fundamental characteristics of a vulnerability. This score can further be refined by the organizations to calculate the bearing of the vulnerability on their environment. The purpose of CVSS is to provide a standard way to measure severity of vulnerabilities therefore CVSS version 2.0 calculator contributes less in proposing the solutions to mitigate the effects of vulnerability on a user environment. The growing number of vulnerabilities requires to have more than a simple CVSS calculator that can also propose the remediation actions for the organizations. This research paper reports on the functionality of previously developed software application to enhance the functionalities of standard CVSS version 2.0 calculator. The developed software application is capable of proposing the optimum remedial actions against vulnerabilities for organizations, requiring minimal time and efforts. This software application will be freely available for use.
\end{abstract}

\section{Keywords}

CVSS, vulnerability scoring system, environmental metrics, enhanced CVSS v2 calculator.

\section{Introduction}

The CVSS has become a de-facto standard for documenting the major characteristics of vulnerabilities and measuring potential impacts of vulnerability exploitation [1],[2],[3]. Initially introduced by the National Infrastructure Advisory Council, CVSS is currently being managed by the CVSS Special Interest Group (CVSS-SIG), and the Forum of Incident Response and Security Teams (FIRST). The National Vulnerability Database (NVD) [4] of the U.S. government is a repository of data of more than forty thousand known software vulnerabilities and their base CVSS scores.

The base CVSS score provided by NVD to a vulnerability cannot be used to prioritize the vulnerability remediation activities. The base score serves as a foundation by providing the innate attributes of vulnerability. The CVSS base score always has the largest bearing on the overall CVSS score of a vulnerability. The CVSS however defines equations for temporal and environmental data that can be used to calculate the overall CVSS score that would estimate more precisely the exploitability and impacts of known vulnerabilities to an organization's IT environment. The NVD also provides a simple online CVSS calculator to calculate these overall scores. While the CVSS calculator is a useful tool to obtain a high level understanding of how the CVSS scoring system works, the calculator has not been designed to calculate the possible overall CVSS scores for a range of possible temporal and environmental data. The CVSS environmental metric group values alone can be arranged in up to 1920 combinations for any particular base score value. However, the existing CVSS calculator calculates the overall CVSS score for only one combination of 
environmental metric group values at a time. Therefore, the an optimum combination of environmental characteristics to reduce the CVSS score of a vulnerability to an acceptable level has been, in general, a daunting task.

The following section of the paper discusses the functionality of the software application [1] developed by our team to enhance the functionality of CVSS v2 calculator. The software application was developed to help to make more informed decisions in a timely manner by presenting the best possible scenarios to mitigate the effects of a vulnerability. The security management of an organization usually has limited budget and limited time to take measures to mitigate the effects of vulnerabilities. Accurate and timely decisions help in the proper budget expenditure and reduce the cost of remediation initiatives.

\section{Related work}

Assad Ali et al developed a software application [1] to calculate and analyze the range of overall CVSS scores of a given vulnerability. This application significantly enhances the functionality of CVSS v2.0 calculator, that makes it to calculate the overall CVSS score for all the possible 1920 user environments. This software application requires minimal effort, and filters provided in this application help the user in selecting the best possible environment that is least affected by that vulnerability. Furthermore, this application works as standard CVSS v2.0 calculator. This application reduces the time spent to calculate the overall CVSS score for all the 1920 user environments and is as accurate as standard CVSS v2.0 calculator.

A number of industry and regulatory efforts have been devoted to scoring of vulnerabilities and mitigating the exploitability and impacts of the vulnerabilities. A compliance with the Payment Card Industry Data Security Standard requires system components not to have any vulnerability with CVSS scores of 4.0 and greater [5].

Scarfone and Mell [2] analyzed the base score component of the CVSS v2 scoring system. They identified 702 possible combinations of the base score metric values and analyzed theoretical scoring distributions for the impact and exploitability subscores that correspond to 75 possible base scores in the CVSS v2. However, the focus of this research did not consider the temporal and environmental components of the scoring system.
Frühwirth and Männistö [6] in their research proposed that vulnerabilities cannot be prioritized based on the base CVSS score given by NVD to any vulnerability. Organizations are supposed to add contextual information, so the vulnerabilities can be properly prioritized and response process can be selected for them. In this research the researchers proposed that this can be achieved by using temporal and environmental metrics. They presented a methodology which enables the organization to decide whether investing in improved vulnerability prioritization is worth the costs. The software application developed by our team is believed to simplify this kind of analysis.

A team of NIST professionals discussed in [7] the functionality of CVSS scoring system, and highlighted the importance of environmental metrics while calculating the CVSS score for any particular organization.

Houmb and Franqueira [8] proposed a Target of Evaluation (ToE) risk level estimation model. They estimated misuse frequency from the attributes in the base and temporal metrics, and misuse impact from the attributes in the base and environmental metrics of CVSS. To achieve this purpose they rearranged the CVSS metrics and derived the risk level of a ToE. Their research was focused on vulnerabilities on an operational level and impacts of the vulnerabilities on strategic level.

The impact of three out of the five environmental metrics on CVSS scores has been recently studied in [9]. The author concludes that environmental score calculation is subject to certain difficulties and it is not yet proven

\section{Effects of environmental metric group on CVSS score}

The NVD uses base metric group values of CVSS to generate scores for the potential impact of known vulnerabilities. There are 1920 maximum possible user environments that can be made by the CVSS environmental metric group values. Each of these environment has different affect of the same vulnerability. Although CVSS base score has the highest bearing on the overall CVSS score, but depending on the different requirements of different organizations, CVSS environmental score changes considerably in different user environments. For this reason the CVSS provides two context aware metric groups, the temporal metric group and the environmental metric group, for the estimation of 
overall vulnerability scores corresponding to various IT environments.

Values of the environmental metric group change the degree of potential impacts and exploitability of vulnerabilities in the various IT environments. It is up to the organization's incident prevention management to control the risks and estimate the overall CVSS score, also known as environmental CVSS score, by invoking environmental metric group values of the CVSS. The overall score is the score for a particular vulnerability in a particular user environment which allows estimating the risks posed by the vulnerability to the organization.

The environmental metric group measures three different characteristics of a vulnerability: (1) Collateral Damage Potential, which measures the loss in terms of life, physical assets, revenue and productivity if a particular vulnerability is exploited; (2) Target Distribution, which measures the percentage of the systems in a user environment being affected by the vulnerability, and lastly (3) the three security requirement metrics, which measure the significance of three security requirements, i.e. Confidentiality Requirement, Integrity Requirement, and Availability Requirement. Depending on criticality of the information assets, an organization's incident prevention and risk management team can assign any amount of importance to these security requirements, while higher security requirements in general lead to higher overall CVSS scores.

\section{Effects of environmental metric group values and limitation of overall CVSS score}

The CVSS provides an authentic and accurate score to each vulnerability found and is a standardized method for rating IT vulnerabilities. A vulnerability cannot be prioritized on the basis of CVSS base score alone. Each vulnerability needs to be made contextual by invoking the environmental metrics group values of CVSS. Hence overall CVSS score generated will reflect the severity of the vulnerability in a particular user environment. Simulations reported in [1], [2], [6], [9], and also our own simulations confirmed that the environmental contextual factors can significantly modify the base score and provide a range of possible overall CVSS scores. The CVSS environmental metric group allows 1920 different combinations of environmental values for any given vulnerability, i.e. for a single base score value. There is a many-to-one relationship between possible combinations of the environmental score metric values and the overall CVSS score, so distinct combinations of environmental metric group values can generate the same overall CVSS score value. Depending on the business and other obligatory requirements, the management of the would be affected organization may decide to take remedial actions.

The CVSS v2 calculators were not designed to be tools for optimization during the risk assessment process. CVSS v2 calculator was designed to measure the score of one vulnerability on one user environment at a time. Which remedial action should be taken to minimize the overall CVSS score for a user environment lies beyond the capabilities of standard CVSS v2 calculator. Therefore, the selection of an acceptable environment is, from a risk management perspective, a tedious task. The enhanced CVSS v2 calculator which makes the risk mitigation process more accurate and less time consuming is described in Section 5.

Incident prevention management teams typically calculate the set of overall scores, and the results are then compiled and compared to decide which remedial actions would reduce the impact, exploitability and overall CVSS score in an efficient way. Ideally, this requires the team to compute, for each given vulnerability, the overall CVSS score for every combination of possible environmental metric group values, and then to arrange those computed results in a useful way. This process using the currently available CVSS calculator requires a lot of time and effort, and thus slow down the remediation activities.

\section{Software tool for studying effects of CVSS metric group values on overall score}

The standard CVSS v2 calculator was not designed to help the user organizations in taking any remedial action against vulnerabilities. The software application [1] developed further enhanced the capabilities of standard CVSS v2 calculator. This application not only helps in making more informed decisions, but also minimized the time and efforts to identify the optimum combination to reduce the overall score to an acceptable level.

This enhanced CVSS calculator uses the same scoring equations and metric values used by the 
standard CVSS v2 calculator. Therefore the score values generated by the enhanced CVSS calculator are similar to score values generated by the standard CVSS v2 calculator. The rounding off problem identified in [10] was minimized to possible extent.

The enhanced CVSS v2 calculator has two Tab pages. The Tab page 1 is similar to standard CVSS v2 calculator that is used to measure the potential exploit of the vulnerability in the user environment. The Tab page 2 is the added functionality which presents all the possible environments and overall CVSS score values for each environment in a grid. Tab page 2 also shows all the CVSS score values, subscore values and current environmental metric group values of the user environment. The grid allows the user to arrange the calculated score values in ascending/descending order of overall CVSS score or any environmental metric group value. The filters provided in the grid headers will enable to see the possible user environments and overall CVSS score generated while keeping the certain environmental metric value(s) unchanged.

The Payment Card Industry (PCI) Data Security Standard requires that compliant organizations must use the CVSS base score for the severity level and "to be considered compliant, a component must not contain any vulnerability that has been assigned a CVSS base score equal to or higher than 4.0" [11]. "Anti-virus software must be used on all the systems generally affected by the malware to protect systems from current and evolving malicious software threats” [12].

Example: The NVD entry [13] CVE-2010-0111 describes the vulnerability in Symantec Antivirus Corporate Edition (SAVCE) 10.x. A remote user can cause denial of service conditions and can execute programs on the target user's system [14]. It is a highrisk vulnerability with base score of 9.3, impact subscore 10.0 and exploitability subscore 8.6. This vulnerability or one such vulnerability, if the software is used can jeopardize any organization.

To mitigate the risks posed by the given vulnerability, the enhanced CVSS v2 calculator [1] would suggest, depending on user preferences, up to 1920 different possible combinations of environmental metrics or possible user environments, generating overall CVSS scores from 0.0 to 9.6 having 34 different overall CVSS score values, as shown in Figure 1.

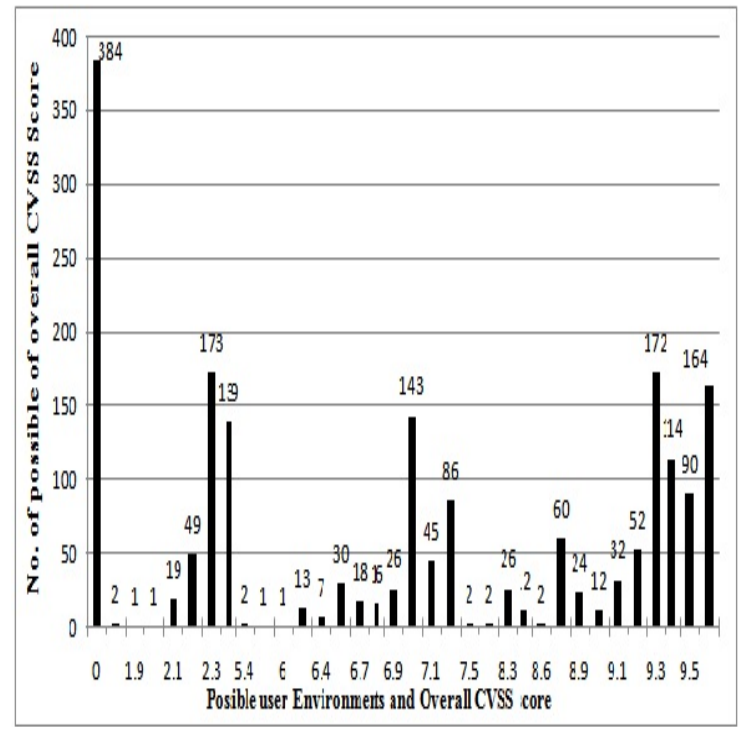

Figure 1. Possible overall CVSS score values for CVE-2010-0111vulnerability.

The organizations that might be affected by the given vulnerability have 32 different choices of overall CVSS scores to reduce the affects of the vulnerability on their environment. This information when converted into pie-chart for the given high level vulnerability shows that there are 114 possible user environments having medium level severity i.e. 5.9\% of the possible user environments and 1038 with low level severity i.e. $40 \%$ of the possible user environments, as shown in Figure 2.

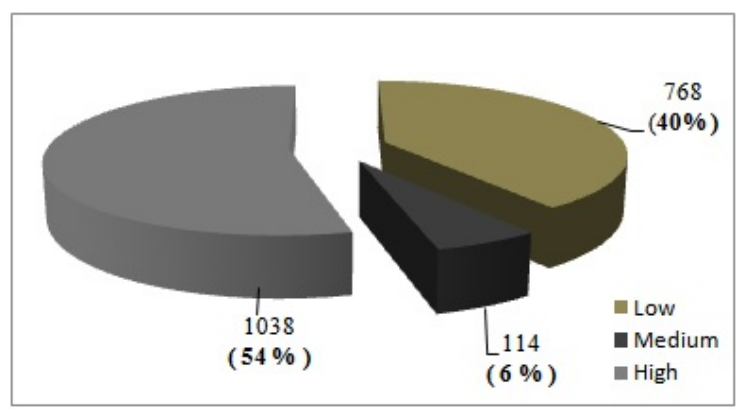

Figure 2. Possible user environments w.r.t. CVSS severity ranking.

Some business requirements may not allow the security management of an organization to make changes in certain environmental metrics or allow them to make changes up to certain level. They then 
can select the metric values of the required metrics from the dropdown menus in the enhance CVSS v2 calculator. This will show the remaining choices of possible user environments to reduce the severity of the vulnerability.

\section{Conclusion}

Mitigation of the affects of vulnerabilities is the foremost objective of the security management of an organization. Due to the large number of possible combinations of values of temporal and environmental metric values, the selection of optimum environmental characteristics has been a difficult and time consuming process. Time plays a very important role in vulnerabilities mitigation. Accurate and timely mitigation of the vulnerabilities can not only reduce the severity of the vulnerabilities, but can also help organizations to meet certain business standards. The practical software tool expands the functionality of available CVSS calculators in the way that makes the estimation of possible overall CVSS scores manageable, and is expected to assist organizations in making informed risk management decisions. This software application will be freely available to organizations.

\section{Acknowledgement}

The first author would like to thank Mazhar Mahmood, Akhtar Ali Qureshi and Vivek Verma for their interest, stimulating discussions and support.

\section{References}

[1] Assad Ali, Pavol Zavarsky, Dale Lindskog, Ron Ruhl, "A Software Application to Analyze the Effects of Temporal and Environmental Metrics on Overall CVSS v2 Score," in Proceeding of WorldCIS-2011 Conference. London, UK, 2011, pp. 123-127.

[2] K. Scarfone and P. Mell, "An Analysis of CVSS Version 2 Vulnerability Scoring,” National Institute of Standards and Technology (NIST), Oct. 2009.

[3] P. Mell, K. Scarfone, S. Romanosky, "CVSS: A Complete Guide to the Common Vulnerability Scoring System Version 2.0”, National Institute of Standards and Technology, June 2006.

[4] National Vulnerability Database version 2.2, National Institute of Standards and Technology (NIST), http://nvd.nist.gov/home.cfm (27 November, 2010).
[5] Payment Card Industry Data Security Standard (PCI DSS): Technical and Operational Requirements for Approved Scanning Vendors, Sept. 2006, https:// www.pcisecuritystandards.org/pdfs/pci_dss_technical _and_operational_requirements_for_approved_scanni ng_vendors_ASVs_v1-1.pdf, (27 November, 2010).

[6] Ch. Frühwirth and T. Männistö, "Improving CVSSbased vulnerability prioritization and response with context information," Helsinki University of Technology, Finland, Oct. 2009.

[7] M. R. Chandramouli, T. Grance, R. Kuhn, and S. Landau," Common Vulnerability Scoring System," IEEE Computer Society, November 2006.

[8] S. H. Houmb and V. N. L. Franqueira, "Estimating ToE Risk Level using CVSS,” Information Systems Group, CTIT, University of Twente, Drienerlolaan, Enschede, The Netherlands, March 2009.

[9] L. Gallon, "On the impact of environmental metrics on CVSS score," The Second IEEE International Conference on Privacy, Security, Risk and Trust, August 2010, Minneapolis, Minnesota, USA.

[10] M. Terada, "Recurring decimal issue in CVSS v2.0 calculator”, Information-Technology Promotion Agency, Japan, January 2010.

[11] Payment Card Industry (PCI) Data Security Standard, Technical and Operational Requirements for Approved Scanning Vendors (ASVs), Version 1.1, September 2006 https://www. pcisecurit ystandards. org/pdfs/pci_dss_technical_and_operational_requirem ents_for_approved_scanning_vendors_ASVs_v11.pdf.

[12] Payment Card Industry (PCI) Data Security Standard, Requirements and Security Assessment Procedures, Version 2.0, October 2010, https://www. Pcisecurity standards.org/documents/pci_dss_v2.pdf, (27 March, 2011).

[13] National Vulnerability Database, "Vulnerability Summary for CVE-2010-0111,” http://web.nvd .nist. gov/view/vuln/detail?vulnId=CVE-2010-0111, (27 March, 2011).

[14] Security Tracker Archives, Security Tracker Alert ID: 1024997, http://securitytracker.com/id/1024997, (27 March, 2011). 